\title{
Long-Term Type 1 Diabetes Enhances In-Stent Restenosis after Aortic Stenting in Diabetes-Prone BB Rats
}

\author{
Geanina Onuta, ${ }^{1}$ Hendrik C. Groenewegen, ${ }^{2}$ Flip A. Klatter, ${ }^{1}$ Mark Walther Boer, ${ }^{1}$ \\ Maaike Goris, ${ }^{2}$ Harry van Goor, ${ }^{3}$ Anton J. M. Roks, ${ }^{4}$ Jan Rozing, ${ }^{1}$ Bart J. G. L. de Smet, ${ }^{2}$ \\ and Jan-Luuk Hillebrands ${ }^{3}$
}

${ }^{1}$ Section of Immunology, Department of Cell Biology, University Medical Center Groningen, University of Groningen, 9700 RB Groningen, The Netherlands

${ }^{2}$ Department of Cardiology, Thorax Center, University Medical Center Groningen, University of Groningen, 9700 RB Groningen, The Netherlands

${ }^{3}$ Division of Pathology, Department of Pathology and Medical Biology, University Medical Center Groningen, University of Groningen, 9700 RB Groningen, The Netherlands

${ }^{4}$ Section of Pharmacology and Vascular and Metabolic Disease, Department of Internal Medicine, Erasmus Medical Centre, 3015 CE Rotterdam, The Netherlands

Correspondence should be addressed to Jan-Luuk Hillebrands, j.l.hillebrands@path.umcg.nl

Received 13 September 2010; Revised 9 December 2010; Accepted 4 January 2011

Academic Editor: Monica Fedele

Copyright ( $) 2011$ Geanina Onuta et al. This is an open access article distributed under the Creative Commons Attribution License, which permits unrestricted use, distribution, and reproduction in any medium, provided the original work is properly cited.

Type 1 diabetic patients have increased risk of developing in-stent restenosis following endovascular stenting. Underlying pathogenetic mechanisms are not fully understood partly due to the lack of a relevant animal model to study the effect(s) of long-term autoimmune diabetes on development of in-stent restenosis. We here describe the development of in-stent restenosis in long-term ( $\sim 7$ months) spontaneously diabetic and age-matched, thymectomized, nondiabetic Diabetes Prone BioBreeding (BBDP) rats $(n=6-7$ in each group). Diabetes was suboptimally treated with insulin and was characterized by significant hyperglycaemia, polyuria, proteinuria, and increased $\mathrm{HbA}_{1 \mathrm{c}}$ levels. Stented abdominal aortas were harvested 28 days after stenting. Computerized morphometric analysis revealed significantly increased neointima formation in long-term diabetic rats compared with nondiabetic controls. In conclusion, long-term autoimmune diabetes in BBDP rats enhances in-stent restenosis. This model can be used to study the underlying pathogenetic mechanisms of diabetes-enhanced in-stent restenosis as well as to test new therapeutic modalities.

\section{Introduction}

In-stent restenosis (ISR) is the most common complication associated with coronary stenting and is histologically characterized by occlusive neointima formation. As yet, no adequate treatment modalities are available to treat or prevent development of ISR [1-3]. The use of drugeluting stents has significantly reduced the incidence of ISR when compared with bare metal stents, but also resulted in increased rates of late stent thrombosis [4]. Diabetes mellitus (DM) has been associated with increased risk to develop (in-stent) restenosis both after percutaneous transluminal coronary angioplasty as well as coronary stenting [5-14]. $\mathrm{DM}$ is a risk factor for ISR development after using both drug-eluting and bare metal stents as revealed by various meta-analyses (primarily including type 2 diabetic patients) [15-17]. The beneficial effects on ISR of drug-eluting stents over bare metal stents as observed in non-diabetic patients appear to be less clear in diabetic patients. Although the exact pathogenetic mechanism underlying increased ISR development in diabetic patients is as yet unknown, it at least appears to be due to an exaggerated neointimal response after coronary stent placement as determined by intravascular ultrasound [18]. Many studies thus favor for a deleterious 
effect of DM on the development of ISR, although the precise magnitude of this effect is not clear. Especially age may act as a confounder as older age is associated with increased restenosis rates independent of DM [17].

To study the molecular and cellular mechanism(s) underlying DM-enhanced development of ISR, clinically relevant animal models may be of great value. However, despite the increasing numbers of diabetic patients worldwide together with coronary artery disease being a significant source of morbidity and mortality in these patients, relevant animal models to study ISR are scarce. Although the Zucker diabetic fatty (ZDF) rat has been used as a model to study type 2 DM-associated restenosis [19-21], a reliable model to study the long-term effects of type 1 diabetes on ISR development is still lacking. Whereas type $1 \mathrm{DM}$ represents only $5-10 \%$ of all diabetics it may be associated with severe coronary artery disease at a relatively young age as demonstrated in a selected population of type 1 diabetics eligible for kidney and/or pancreas transplantation [22]. Various rodent models of type $1 \mathrm{DM}$ have been used to study the effects of diabetes on mechanically induced restenosis in mice, rats, or rabbits, in which diabetes is chemically induced using streptozotocin or alloxan and in which contradicting results were obtained [23]. In addition to rodent models, also a streptozotocin-induced diabetes porcine model has been used to study the development of ISR. In this model, a high mortality rate $(\sim 45 \%)$, a relatively short course of diabetes (12 weeks), and most likely high costs may actually hamper extensive use of this model [24]. Both streptozotocin and alloxan are toxic compounds sharing structural similarities with glucose which explains their selective uptake in cells expressing the GLUT2 glucose transporter. As pancreatic ßcells have relatively high levels of GLUT2, streptozotocin and alloxan are relatively, but not solely, toxic to B-cells. Because of the possible toxic side effects of these chemicals and to model more accurately human type 1 (autoimmune) $\mathrm{DM}$, the availability of a spontaneous type $1 \mathrm{DM}$ model for the development of (in-stent) restenosis is needed. To this end, we here describe the use of inbred Diabetes Prone BioBreeding (BBDP/Wor) rats as a model to study diabetesenhanced development of ISR. Inbred BBDP rats are derived from a Canadian colony of outbred Wistar rats (i.e., the BB Wistar rat) in which diabetes developed spontaneously in the 1970s [25]. BBDP/Wor rats develop autoimmune diabetes spontaneously due to severe lymphopenia and preferential lack of immunoregulatory $\mathrm{T}$ cells [26-28]. Using the rat abdominal aorta stenting model described previously [29], we tested the hypothesis that long-term suboptimally treated hyperglycaemic BBDP rats develop enhanced ISR compared with non-diabetic age-matched control rats.

\section{Materials and Methods}

2.1. Rats. Specified pathogen-free Diabetes Prone BB (BBDP/Wor) and Diabetes-Resistant (BBDR/Wor) rats were bred at the Central Animal Facility of the University Medical Center Groningen, University of Groningen, Groningen, The Netherlands. Original breeding stocks were obtained from
Biomedical Research Models (BRM Inc., Worcester, MA, USA). Rats were kept under clean conventional conditions and were fed standard rat chow and acidified water ad libitum. All animals received humane care in compliance with the Principles of Laboratory Animal Care (NIH Publication no. 85-23, revised 1996) and the Dutch Law on Experimental Animal Care.

2.2. Diabetes Development in BBDP Rats. In the BBDP/Wor colony maintained at the University Medical Center Groningen, $80-90 \%$ of the rats spontaneously develop diabetes from $\sim 70$ days of age. Rats were considered diabetic when blood glucose levels exceeded $15 \mathrm{mmol} / \mathrm{L}$ as measured in peripheral blood obtained by tail vein puncture and by using a glucose sensor (Accu-Chek Sensor Comfort, Roche Diagnostics Nederland B.V., Almere, The Netherlands). In this study, 9 recent onset diabetic BBDP rats were initially included (both male and female) which received a 1/2 ( 3-4 mm length) Linplant sustained release insulin implant (LinShin Canada Inc, Toronto, ON, Canada) subcutaneously at diagnosis using a trocar. According to the manufacturer the implants had an estimated insulin release of $\sim 1 \mathrm{U} / 24 \mathrm{~h} /(1 / 2)$ implant for $>40$ days. We aimed at maintaining the blood glucose values in insulin-treated diabetic rats between $15-20 \mathrm{mmol} / \mathrm{L}$ during the entire follow-up period (up to 10 months of age). During follow-up diabetic rats were weighed 2-3 times a week. In case of weight loss, blood glucose levels were measured as described above. When blood glucose levels exceeded $20 \mathrm{mmol} / \mathrm{L}$ or blood glucose levels were between $15-20 \mathrm{mmol} / \mathrm{L}$ in the presence of substantial weight loss, rats were reimplanted with an insulin implant as described above.

In addition to the insulin-treated diabetic BBDP rats also a control group of age-matched non-diabetic BBDP rats (initially $n=9$ ) was included. Diabetes development was prevented by performing thymectomy at the age of 21 days as we described in detail elsewhere [30]. Weight and blood glucose levels of thymectomized non-diabetic BBDP rats were measured once every two weeks to obtain basal values.

2.3. $H b A_{1 c}$ Measurements. To determine whether long-term suboptimal insulin treatment of diabetic BBDP rats is associated with increased glycated hemoglobin $\left(\mathrm{HbA}_{1 \mathrm{c}}\right)$ levels indicative of poor glycaemic control, we analyzed $\mathrm{HbA}_{\mathrm{lc}}$ levels in a separate cohort of long-term diabetic BBDP rats $(n=5)$, as well as in spontaneously protected nondiabetic $\mathrm{BBDP}$ rats $(n=2)$ and non-diabetic BBDR/Wor rats $(n=6) . \mathrm{HbA}_{1 \mathrm{c}}$ levels were determined in freshly obtained peripheral blood (tail vein puncture) using the BIO-RAD in 2it A1C analyzer (Bio-Rad Laboratories B.V., Veenendaal, The Netherlands). Values are expressed as DCCT- $\mathrm{HbA}_{1 c} \%$.

2.4. Renal Function Measurements. Two weeks before stenting renal function of the long-term diabetic and nondiabetic BBDP rats was determined by housing the rats in individual, urine-collecting metabolic cages for 24 hours and by collection of blood plasma. Plasma and urine creatinine levels were determined using the enzymatic colorimetric 
TABLE 1: Characteristics of the diabetic (DM) and thymectomized non-diabetic (non-DM) BBDP rats that were stented and evaluated for the development of in-stent restenosis.

\begin{tabular}{|c|c|c|c|c|c|c|}
\hline & $N$ & $\begin{array}{l}\text { Gender } \\
\text { ㅇ/ఠ }\end{array}$ & $\begin{array}{l}\text { Diabetes onset } \\
\text { (median in days) }\end{array}$ & $\begin{array}{l}\text { Age at stenting } \\
\text { (median in days) }\end{array}$ & $\begin{array}{l}\text { Diabetes duration at stenting } \\
\text { (median in days) }\end{array}$ & $\begin{array}{c}\text { Serum creatinine } \\
(\text { mean } \pm \text { SEM in } \mu \mathrm{mol} / \mathrm{L})\end{array}$ \\
\hline Non-DM BBDP & 6 & $4 / 2$ & N.A. ${ }^{b}$ & $298[270-412]^{\mathrm{c}}$ & N.A.b & $51 \pm 7$ \\
\hline DM BBDP & 7 & $3 / 4$ & $82[72-140]^{\mathrm{c}}$ & $270[270-290]^{\mathrm{c}}$ & $198[150-204]^{\mathrm{c}}$ & $56 \pm 9$ \\
\hline
\end{tabular}

${ }^{\mathrm{a}}$ Serum creatinine levels were determined 2 weeks prior to stenting.

${ }^{b}$ N.A.: not applicable.

${ }^{c}$ Values between brackets indicate minimum and maximum values.

assay CREA plus (Roche Diagnostics GmbH, Mannheim, Germany) [31]. Total urinary protein excretion was determined using the Roche Diagnostics Urinary/CSF Protein assay (Roche Diagnostics $\mathrm{GmbH}$, Mannheim, Germany) $[32,33]$.

2.5. Stent Implantation. At a median diabetes duration of 28 weeks (7 months) 9 BBDP rats were stented in the abdominal aorta as described previously [29]. As a control, 9 age-matched, thymectomized non-diabetic BBDP rats were included. Briefly, under anesthesia ( $2 \%$ isoflurane (Abbott, Hoofddorp, The Netherlands), $0.4 \mathrm{~L} / \mathrm{min}_{2}$ and $0.4 \mathrm{~L} / \mathrm{min}$ $\mathrm{N}_{2} \mathrm{O}$ ) the abdominal cavity was opened. The aorta was dissected, and surrounding connective tissue was removed. Next, two vascular clips were placed onto the aorta distal to the renal arteries and proximal to the aortic bifurcation. A small incision was then made in the distal abdominal aorta, and the balloon catheter was inserted and inflated to $9 \mathrm{~atm}$ pressure to deploy a premounted $2.5 \times 9 \mathrm{~mm}$ BeStent 2 bare metal stent (Medtronic-Bakken Research, Maastricht, The Netherlands). After deflation and removal of the balloon, the aortic incision was closed with a 9-0 suture. Reperfusion was established by removing the clips, and the abdomen was closed with 4-0 sutures. Starting 5 days before stenting until the end of the experiment, rats were fed chow containing $0.33 \mathrm{mg}$ clopidogrel/gram chow (Plavix, Sanofi-Aventis, Gouda, The Netherlands) in order to prevent platelet aggregation and to mimick the human clinical setting. Four weeks after stenting, rats were anesthesized and heparinized systemically with 500 IU i.v. (Leo Pharma, Breda, The Netherlands). The stented aortas were harvested, fixed in $4 \%$ formaldehyde, and embedded in methyl metacrylate for further histological analysis. During stenting, 3 non-diabetic control BBDP rats died because of perforation of the aorta, resulting in an overall mortality of $17 \%$. In addition, 2 stents from long-term diabetic BBDP rats were lost during histological processing. Overall, stents from 7 diabetic and 6 non-diabetic BBDP rats were included for histological analysis.

2.6. Quantification In-Stent Restenosis (ISR). To quantify the severity of ISR, computerized morphometric analysis was performed on Lawson (elastin) stained sections obtained from the proximal, middle, and distal parts of each stent. The neointimal area was calculated by measuring the total area within the internal elastic lamina and the remaining lumen using an Olympus BX-50F4 microscope equipped with an Olympus c-3030 zoom digital camera and Olympus DP-Soft version 3.0 software (Olympus, Tokyo, Japan). Total surface neointima (in $\mu \mathrm{m}^{2}$ ) was then calculated by subtracting surface remaining lumen from the total surface area within the internal elastic lamina.

2.7. Quantification Vessel Injury. The mean vessel injury score was determined as described previously [29] using a method originally developed by Schwartz et al. [34]. Vessel injury at every stent strut within a cross-section (9-10 struts/cross-section) was determined based on the anatomic vessel structures penetrated by each strut. This value ranged from 0 (least injury) to 3 (most injury). For each cross-section the mean injury score was calculated. From each stent 6-8 cross-sections (taken from the proximal, middle, and distal parts of the stent) were analyzed. The total mean injury score was expressed as the mean of the injury scores of all cross-sections analyzed within one stent.

2.8. Statistical Analysis. Data are expressed as mean \pm SEM. Differences between two groups were analyzed for statistical significance using an independent samples $t$-test. All $P$ values were two-tailed, and a $P$-value $<.05$ was considered statistically significant. Statistical analyses were performed using GraphPad Prism 5.00 for Windows (GraphPad Software Inc., USA).

\section{Results}

3.1. Glucose Homeostasis in Long-Term Diabetic BBDP Rats. Diabetes incidence in the BBDP colony maintained at the University Medical Center Groningen is $80-90 \%$. Median age of diabetes onset in the BBDP rats $(n=7)$ that were stented and included for histological analysis was 82 days (Table 1). Insulin treatment using insulin-releasing implants resulted in an almost permanent hyperglycaemic state (blood glucose $>8 \mathrm{mmol} / \mathrm{L}$ ) in all diabetic rats. In individual rats blood glucose oscillations were observed that varied between 8 and $27 \mathrm{mmol} / \mathrm{L}$. Depending on the blood glucose levels $(>20 \mathrm{mmol} / \mathrm{L}$ or $15-20 \mathrm{mmol} / \mathrm{L}$ in the presence of substantial weight loss ( $>10 \%$ weight loss compared with previous measurement)), rats received an insulin implant. Time between reimplantations varied between 5 and 35 days. As non-diabetic age-matched controls, 6 thymectomized BBDP rats were included. Figure 1 shows the blood glucose 


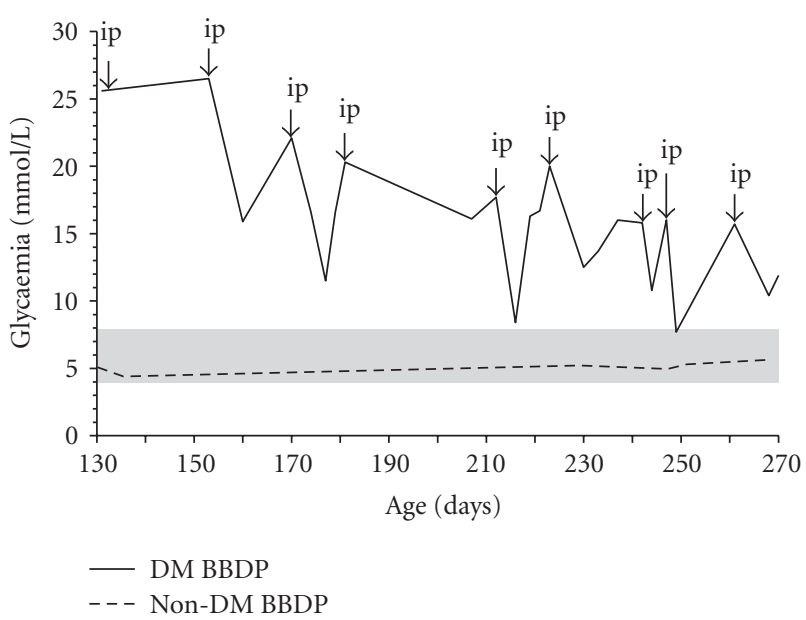

Figure 1: Oscillations in blood glucose levels in a representative diabetic (DM) BBDP rat treated with insulin pellets implanted subcutaneously. During a follow-up period of 140 days the DM BBDP rat received multiple insulin pellet implants (ips) guided by rapid development of hyperglycaemia (solid line) and drop in body weight (not shown). For comparison, glucose levels in an agematched thymectomized non-DM BBDP rat are shown. The grey area indicates the normoglycaemic range (4-8 $\mathrm{mmol} / \mathrm{L})$.

oscillations observed in an individual diabetic and nondiabetic BBDP rat during a time frame of 140 days during which the diabetic rat received 9 insulin implants. The median age at stenting was 270 and 298 days for the diabetic and non-diabetic BBDP rats, respectively (not significantly different), translating in a median diabetes duration of 198 days (Table 1). During the total follow-up period till stenting mean blood glucose level after diabetes onset in diabetic BBDP rats was $15.0 \pm 0.4 \mathrm{mmol} / \mathrm{L}$ compared with $5.3 \pm 0.1 \mathrm{mmol} / \mathrm{L}$ in thymectomized non-diabetic BBDP rats (Figure 2(a), $P<.001$ ). As increased $\mathrm{HbA}_{1 \mathrm{c}}$ levels have been associated with increased cardiovascular risk in human diabetics, we questioned whether long-term diabetes in our $\mathrm{BBDP}$ rat model was also associated with increased $\mathrm{HbA}_{1 \mathrm{c}}$ levels. To this end, $\mathrm{HbA}_{1 \mathrm{c}}$ levels were determined in a separate group of long-term diabetic BBDP rats $(n=5)$ with a mean diabetes duration of $249 \pm 50$ days which was not statistically different from the mean diabetes duration in the stented $\mathrm{BBDP}$ rats (217 \pm 7 days) at sacrifice. For comparison, $\mathrm{HbA}_{1 \mathrm{c}}$ levels were determined in spontaneously protected nondiabetic BBDP rats $(n=2)$ and non-diabetic BBDR/Wor rats $(n=6)$. As shown in Figure 2(b), long-term diabetes resulted in significantly increased $\mathrm{HbA}_{1 \mathrm{c}}$ levels $(11.9 \pm 0.2 \mathrm{DCCT}$ $\left.\mathrm{HbA}_{1 c} \%\right)$ compared with age-matched non-diabetic $\mathrm{BB}$ rats $(5.1 \pm 0.1$ DCCT-HbA $1 c \%)$.

3.2. Renal Function in Long-Term Diabetic BBDP Rats. Two weeks before stenting part of the long-term diabetic and nondiabetic BBDP rats were housed in metabolic cages for $24 \mathrm{hrs}$ to collect urine. Long-term diabetes and the associated permanent hyperglycaemic state resulted in a 6-fold increased $(P<.001)$ urinary volume compared with non-DM BBDP rats (Figure 3(a)). This was accompanied by severe polydipsia (not shown). Furthermore, total urinary protein excretion (Figure 3(b), $P<.01$ ) and urinary creatinine excretion (Figure $3(\mathrm{c}), P<.001$ ) were significantly increased in long-term DM BBDP rats compared with non-DM BBDP rats. Increased urinary creatinine excretion in DM BBDP rats suggests the presence of glomerular hyperfiltration which is commonly observed in diabetics. Hyperfiltration was supported by the slightly increased creatinine clearance rates observed in the long-term $\mathrm{DM}$ rats $(1.7 \pm 0.3 \mathrm{~mL} / \mathrm{min}$ in $\mathrm{DM}$ versus $1.0 \pm 0.2 \mathrm{~mL} / \mathrm{min}$ in non-DM, not significant). Despite the presence of proteinuria and hyperfiltration in long-term DM BBDP rats, plasma creatinine levels in these rats were similar to the levels detected in age-matched nonDM BBDP rats indicating preserved renal function in the presence of diabetes (Table 1). Histological analysis of PASstained renal sections did not reveal increased interstitial and glomerular matrix expansion in long-term DM BBDP rats (not shown).

\subsection{Enhanced In-Stent Restenosis in Long-Term Diabetic} $B B D P$ Rats. In total, 18 rats (9 DM and 9 non-DM BBDP rats) received a bare metal stent (Figure $4(a)$ ) in the abdominal aorta. During the stenting procedure, 3 non-DM BBDP rats died because of perforation of the aorta (mortality rate of 17\%). Age of the DM and non-DM BBDP rats at stenting was similar between both groups (Table 1, median age, resp., 270 and 298 days, not significantly different). Diabetes duration (median) in DM BBDP rats at stenting was 198 days (Table 1). Stents were harvested 28 days after stenting. Stents from 2 DM BBDP rats were lost during histological processing. Finally, stents from $7 \mathrm{DM}$ and 6 nonDM BBDP rats were histologically analyzed for the severity of ISR. At 28 days poststenting in both non-DM and DM BBDP rats development of ISR was detected which was characterized by neointima formation surrounding the stent struts. Figures 4(d), 4(e), 4(f), and 4(g) show representative photomicrographs of ISR in, respectively, non-DM and DM BBDP rats. Quantitative analysis revealed that long-term DM resulted in a $32 \%$ increase in surface neointima compared with non-DM BBDP rats (Figure 4(b), $P=.02$ ). This increase in neointima formation in DM BBDP rats was not associated with an increased mean injury score (Figure 4(c), $P=$.957).

\section{Discussion}

Diabetes mellitus (DM) is associated with increased risk for the development of in-stent restenosis (ISR) [15-17]. Underlying pathogenetic mechanisms are as yet unknown, and adequate treatment modalities are lacking. In order to increase our insights into the molecular and cellular mechanism(s) underlying type $1 \mathrm{DM}$-enhanced development of ISR a clinically relevant rodent model might be of great value. Yet a model as such is not available. We therefore tested the hypothesis that long-term spontaneously diabetic hyperglycaemic BBDP rats develop enhanced ISR and may be used as a suitable model to study the molecular and cellular mechanism(s) involved in DM-enhanced development of ISR. 


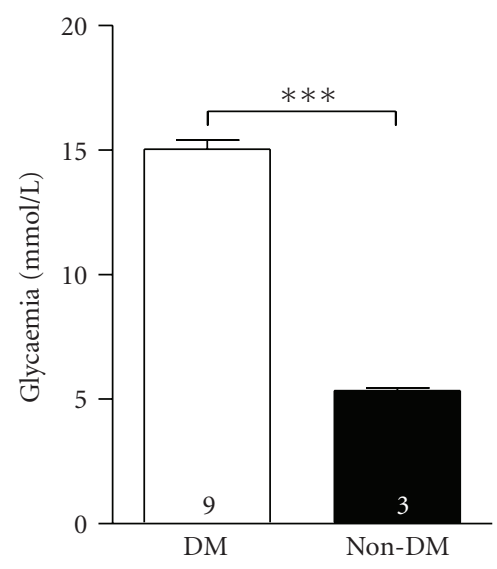

(a)

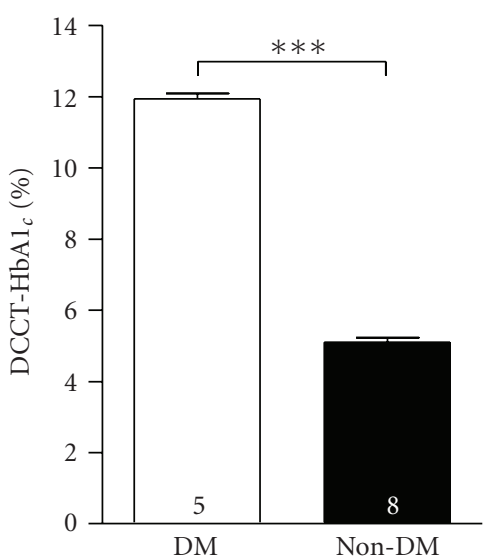

(b)

Figure 2: Blood glucose and $\mathrm{HbA}_{1 \mathrm{c}}$ levels are increased in long-term diabetic (DM) BBDP rats. (a) Average blood glucose level during the entire follow-up period starting at diabetes onset until sacrifice (ranging from 150 to $>200$ days) (white bar). For comparison, mean blood glucose levels in age-matched non-DM BBDP rats are shown (black bar). (b) Hyperglycaemia in long-term diabetic BBDP rats is associated with increased glycated haemoglobin $\left(\mathrm{HbA}_{1 \mathrm{c}}\right)$ levels (white bar) compared with non-DM rats (black bar). Data are expressed as the mean \pm SEM $\left({ }^{* * *} P<.001\right)$. Values within bars indicate the number of rats analyzed. DM: long-term diabetic BBDP rats, Non-DM: non-diabetic rats.

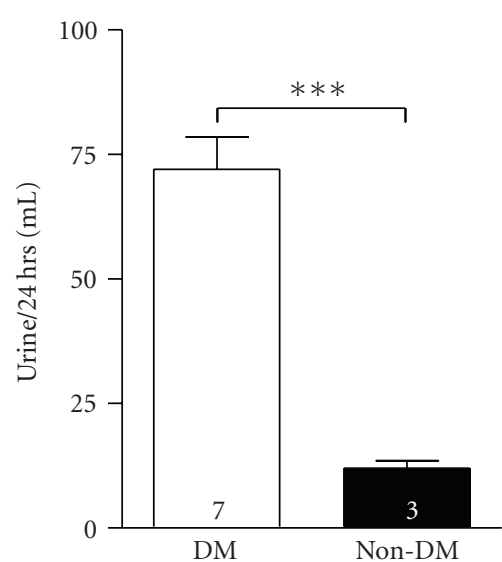

(a)

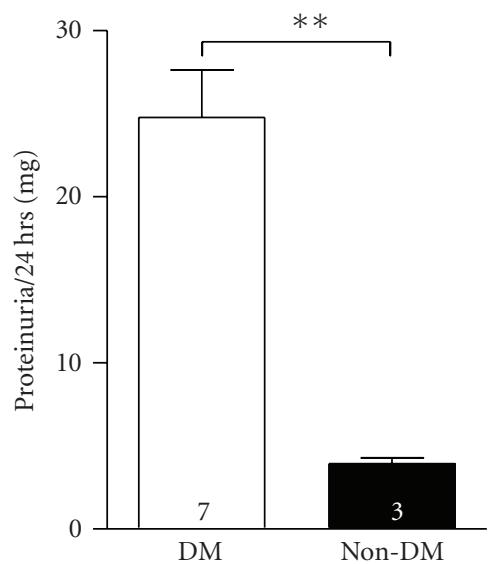

(b)

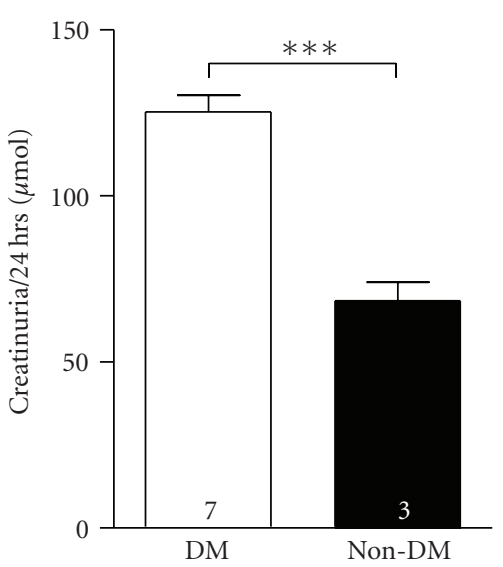

(c)

FIGURE 3: Long-term diabetes in BBDP rats is associated with significant polyuria (a), proteinuria (b), and creatinuria (c). Two weeks prior to stenting rats were housed in metabolic cages, and 24-hour urine samples were collected and analyzed as described in Section 2. Data are expressed as mean $\pm \mathrm{SEM}\left({ }^{* *} P<.01,{ }^{* * *} P<.001\right)$. Values within bars indicate the number of rats analyzed. DM: long-term diabetic BBDP rats, Non-DM: non-diabetic rats.

The hyperglycemic syndrome in BBDP rats develops spontaneously due to a disturbed balance in autoreactive and regulatory $\mathrm{T}$ cells [26-28]. The BBDP rat has been suggested to represent the best rodent model for human type $1 \mathrm{DM}$ as in this model diabetes manifests during adolescence and involves an autoimmune disorder without the need of exogenous intervention.

To achieve suboptimally controlled DM with manifest hyperglycaemia and elevated $\mathrm{HbA}_{1 \mathrm{c}}$ levels, recent onset diabetic BBDP rats were treated with insulin using slow release insulin implants. By doing so, we were able to maintain DM BBDP rats in a rather permanent hyperglycaemic state for at least 8 months. The hyperglycaemic state was associated with increased $\mathrm{HbA}_{1 \mathrm{c}}$ levels as well as polyuria, polydipsia, proteinuria, and glomerular hyperfiltration. Severity of proteinuria was within the range reported by Cohen et al. who also determined protein excretion in long-term DM BBDP rats that were treated with daily insulin injections [35]. As older age is associated with increased restenosis rates independent of DM [17], for our study it was important to include age-matched non-diabetic controls rather than prediabetic young BBDP rats. In order to prevent diabetes development in BBDP rats thymectomy was performed at the age of 21 days as we described previously [30]. DM and non-DM BBDP rats were followed for 7 months after which they received a bare metal stent implanted in the 


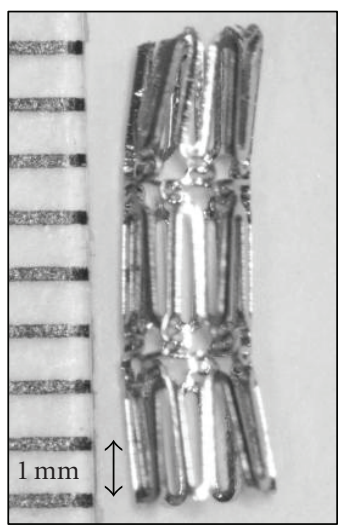

(a)

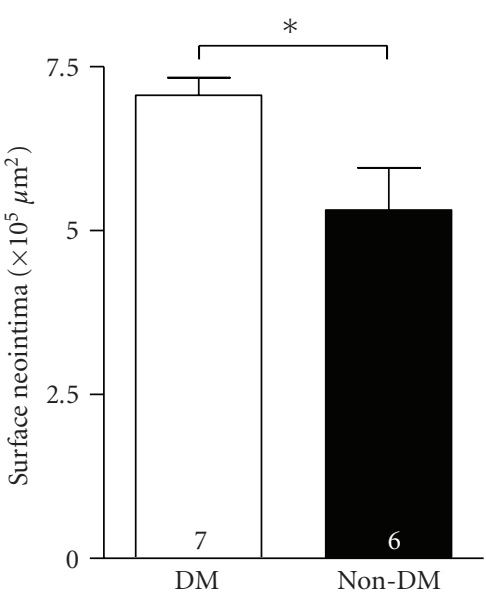

(b)

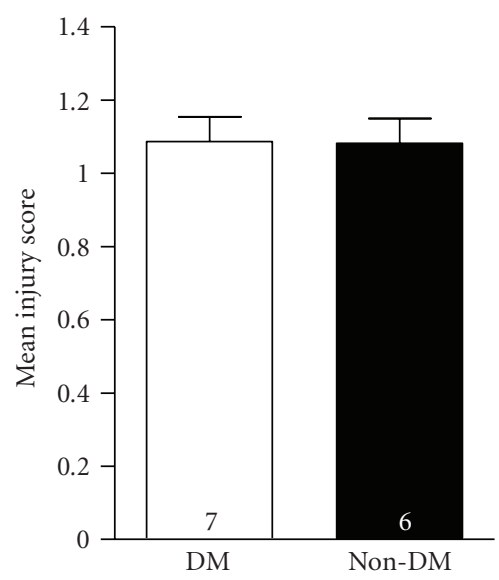

(c)

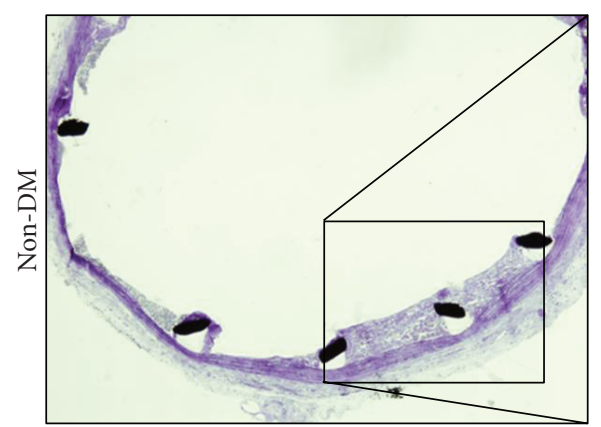

(d)

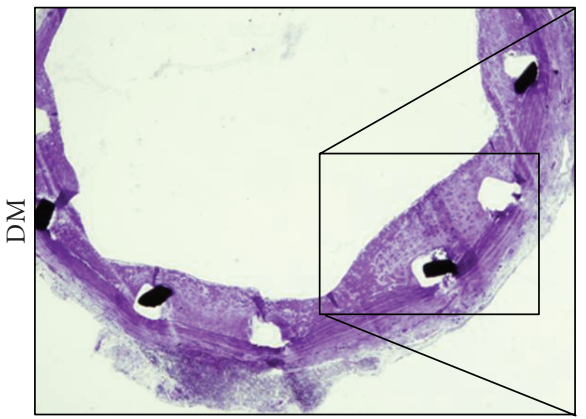

(f)

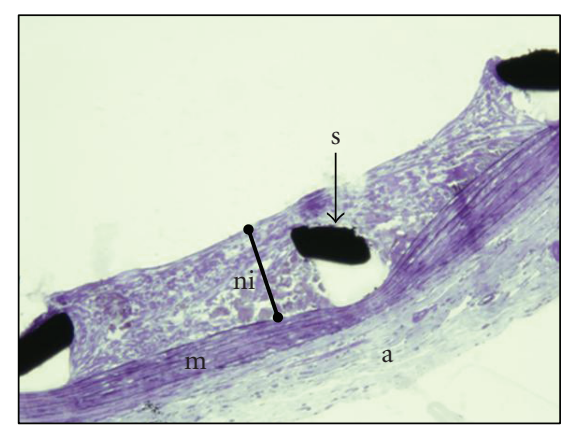

(e)

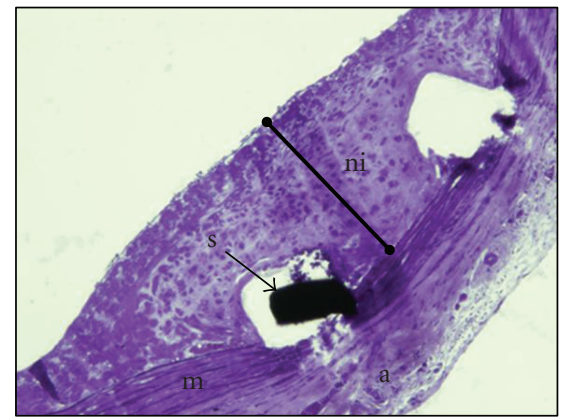

(g)

FIGURE 4: Long-term diabetes in BBDP rats is associated with enhanced in-stent restenosis 4 weeks after stenting in the abdominal aorta. (a) Photograph of a BeStent 2 bare metal stent used for implantation. The inflation balloon has been removed. (b) Surface area neointima present 28 days after stenting in long-term DM (white bar) and non-DM (black bar) BBDP rats. (c) Mean injury score at 28 days after stenting in long-term DM (white bar) and non-DM (black bar) BBDP rats. Data are expressed as the mean \pm SEM $\left({ }^{*} P<.05\right)$. Values within bars indicate the number of rats analyzed. DM: long-term diabetic BBDP rats, Non-DM: non-diabetic rats. ((d), (e)) Representative photomicrographs (Lawson staining) of stented abdominal aortas from a thymectomized non-diabetic (non-DM) BBDP rat 28 days after stenting. ((f), (g)) Representative photomicrographs (Lawson staining) of stented abdominal aortas from a diabetic (DM) BBDP rat 28 days after stenting. (e) and $(\mathrm{g})$ are high-power magnifications (magnification $\times 100)$ of the framed areas shown in, respectively, $(\mathrm{d})$ and $(\mathrm{f})$ (magnification $\times 40)$. a: adventitia; m: media; ni: neointima; s: stent strut.

abdominal aorta. Our results clearly demonstrated that longterm DM significantly enhanced the development of ISR by $32 \%$ compared with non-DM age-matched thymectomized BBDP rats. This enhanced development of ISR in diabetic BBDP rats was not associated with increased vascular injury, that is, increased penetration of the stent struts through the internal elastic lamina into the medial layer. The severity of ISR was previously shown to be positively correlated with the mean injury score [29]. However, in the same study we demonstrated differences in severity of ISR between bare 
metal and sirolimus-eluting stents, which was independent of the mean injury score. Our data suggest that T1DM enhances the development of ISR by factors other than direct vascular injury.

The current study was performed as a proof of concept to demonstrate that long-term T1DM in BBDP rats indeed enhances the development of ISR following stenting in the abdominal aorta. Although we did not study the underlying mechanism(s) of enhanced ISR in diabetic BBDP rats yet, we suggest that long-term diabetes increases the proliferative and migratory capacity of medial and neointimal smooth muscle cells, thereby facilitating neointima formation [36, 37]. In addition, reduced endothelial repair capacity in diabetic BBDP rats might have contributed to enhanced ISR [38], but this needs to be determined.

The major advantage of the BBDP model to study the development of ISR over other T1DM rodent models is the fact that diabetes develops due to autoimmunemediated destruction of pancreatic islets without having the toxic side effects of the use of streptozotocin or alloxan. By using slow release insulin implants we showed that long-term studies are feasible without the need of daily insulin injections. A possible weakness of the model is that BBDP rats are $\mathrm{T}$ cell lymphopenic and are, in that respect, not fully immunocompetent. However, despite $\mathrm{T}$ cell lymphopenia BBDP rats do develop autoimmune diabetes which is mediated by autoreactive $\mathrm{T}$ cells indicating that functional $\mathrm{T}$ cells are present in BBDP rats. Whether $\mathrm{T}$ cells are pivotal in the development of ISR is however unclear. Percutaneous transluminal coronary angioplasty has been shown to induce $\mathrm{T}$ cell activation in a small cohort of 10 patients with stable angina. Patients that developed restenosis had higher $\mathrm{T}$ cell activation levels than patients that did not develop restenosis [39]. However, treatment with the calcineurin inhibitor cyclosporine to prevent $\mathrm{T}$ cell activation did not reduce the development of restenosis in rabbits [40]. We therefore assume that the presence of $\mathrm{T}$ cell lymphopenia in BBDP rats has not been of major influence on the development of ISR.

\section{Conclusions}

The BBDP rat model for type 1 diabetes is suitable for studies on the long-term effects of hyperglycaemia on the development of ISR. Long-term diabetes significantly increased the development of ISR. To our opinion, future studies aiming at the identification of the molecular and cellular mechanisms involved as well as on testing the efficacy of novel therapeutic interventions in this model are warranted.

\section{Acknowledgment}

This work was supported by the Netherlands Organization for Scientific Research (NWO VENI Grant 916.46.104 to J.Luuk Hillebrands) and the Ubbo Emmius Foundation (to G. Onuta). The authors would like to thank Annemieke Smitvan Oosten, Anthony van Dijk, and Manon van Riezen for excellent (bio)technical assistance.

\section{References}

[1] N. A. Scott, "Restenosis following implantation of bare metal coronary stents: pathophysiology and pathways involved in the vascular response to injury," Advanced Drug Delivery Reviews, vol. 58, no. 3, pp. 358-376, 2006.

[2] H. C. Lowe, S. N. Oesterle, and L. M. Khachigian, "Coronary in-stent restenosis: current status and future strategies," Journal of the American College of Cardiology, vol. 39, no. 2, pp. 183-193, 2002.

[3] A. K. Mitra and D. K. Agrawal, "In stent restenosis: bane of the stent era," Journal of Clinical Pathology, vol. 59, no. 3, pp. 232-239, 2006.

[4] S. Garg and P. W. Serruys, "Coronary stents: current status," Journal of the American College of Cardiology, vol. 56, no. 10, supplement 1, pp. S1-S42, 2010.

[5] A. Abizaid, R. Kornowski, G. S. Mintz et al., "The influence of diabetes mellitus on acute and late clinical outcomes following coronary stent implantation," Journal of the American College of Cardiology, vol. 32, no. 3, pp. 584-589, 1998.

[6] C. Berry, J. C. Tardif, and M. G. Bourassa, "Coronary heart disease in patients with diabetes-part II: recent advances in coronary revascularization," Journal of the American College of Cardiology, vol. 49, no. 6, pp. 643-656, 2007.

[7] M. Loutfi, N. T. Mulvihill, M. Boccalatte, B. Farah, J. Fajadet, and J. Marco, "Impact of restenosis and disease progression on clinical outcome after multivessel stenting in diabetic patients," Catheterization and Cardiovascular Interventions, vol. 58, no. 4, pp. 451-454, 2003.

[8] A. Kastrati, A. Schömig, S. Elezi et al., "Predictive factors of restenosis after coronary stent placement," Journal of the American College of Cardiology, vol. 30, no. 6, pp. 1428-1436, 1997.

[9] S. Elezi, A. Kastrati, J. Pache et al., "Diabetes mellitus and the clinical and angiographic outcome after coronary stent placement," Journal of the American College of Cardiology, vol. 32, no. 7, pp. 1866-1873, 1998.

[10] R. Seabra-Gomes, "Percutaneous coronary interventions with drug eluting stents for diabetic patients," Heart, vol. 92, no. 3, pp. 410-419, 2006.

[11] S. G. Ellis and C. R. Narins, "Problem of angioplasty in diabetics," Circulation, vol. 96, no. 6, pp. 1707-1710, 1997.

[12] J. P. Carrozza Jr., R. E. Kuntz, R. F. Fishman, and D. S. Baim, "Restenosis after arterial injury caused by coronary stenting in patients with diabetes mellitus," Annals of Internal Medicine, vol. 118, no. 5, pp. 344-349, 1993.

[13] S. R. Wilson, B. A. Vakili, W. Sherman, T. A. Sanborn, and D. L. Brown, "Effect of diabetes on long-term mortality following contemporary percutaneous coronary intervention: analysis of 4284 cases," Diabetes Care, vol. 27, no. 5, pp. 1137-1142, 2004.

[14] P. K. Kuchulakanti, R. Torguson, D. Canos et al., "Impact of treatment of coronary artery disease with sirolimus-eluting stents on outcomes of diabetic and nondiabetic patients," American Journal of Cardiology, vol. 96, no. 8, pp. 1100-1106, 2005.

[15] C. Stettler, S. Allemann, M. Egger, S. Windecker, B. Meier, and P. Diem, "Efficacy of drug eluting stents in patients with and without diabetes mellitus: indirect comparison of controlled trials," Heart, vol. 92, no. 5, pp. 650-657, 2006.

[16] A. J. Scheen and F. Warzée, "Diabetes is still a risk factor for restenosis after drug-eluting stent in coronary arteries," Diabetes Care, vol. 27, no. 7, pp. 1840-1841, 2004. 
[17] J. Gilbert, J. Raboud, and B. Zinman, "Meta-analysis of the effect of diabetes on restenosis rates among patients receiving coronary angioplasty stenting," Diabetes Care, vol. 27, no. 4, pp. 990-994, 2004.

[18] R. Komowski, G. S. Mintz, K. M. Kent et al., "Increased restenosis in diabetes mellitus after coronary interventions is due to exaggerated intimal hyperplasia: a serial intravascular ultrasound study," Circulation, vol. 95, no. 6, pp. 1366-1369, 1997.

[19] R. Takeda, E. Suzuki, H. Satonaka et al., "Blockade of endogenous cytokines mitigates neointimal formation in obese Zucker rats," Circulation, vol. 111, no. 11, pp. 13981406, 2005.

[20] J. Shelton, D. Wang, H. Gupta, J. M. Wyss, S. Oparil, and C. R. White, "The neointimal response ot endovascular injury is increased in obese Zucker rats," Diabetes, Obesity and Metabolism, vol. 5, no. 6, pp. 415-423, 2003.

[21] A. C. McMahon, H. Zreiqat, and H. C. Lowe, "Carotid artery stenting in the Zucker rat: a novel, potentially 'diabetesspecific' model of in-stent restenosis," Diabetes and Vascular Disease Research, vol. 5, no. 2, pp. 145-146, 2008.

[22] S. Dickinson, T. Rogers, B. Kasiske et al., "Coronary artery disease in young women and men with long-standing insulindependent diabetes," Angiology, vol. 59, no. 1, pp. 9-15, 2008.

[23] H. Gonzalez-Navarro, D. J. Burks, and V. Andres, "Murine models to investigate the influence of diabetic metabolism on the development of atherosclerosis and restenosis," Frontiers in Bioscience, vol. 12, pp. 4439-4455, 2007.

[24] A. J. Carter, L. Bailey, J. Devries, and B. Hubbard, "The effects of uncontrolled hyperglycemia on thrombosis and formation of neointima after coronary stent placement in a novel diabetic porcine model of restenosis," Coronary Artery Disease, vol. 11, no. 6 , pp. $473-479,2000$.

[25] E. B. Marliss, A. F. Nakhooda, P. Poussier, and A. A. F. Sima, "The diabetic syndrome of the 'BB' Wistar rat: possible relevance to type 1 (insulin-dependent) diabetes in man," Diabetologia, vol. 22, no. 4, pp. 225-232, 1982.

[26] J. L. Hillebrands, B. Whalen, J. T. J. Visser et al., "A regulatory $\mathrm{CD}^{+} \mathrm{T}$ cell subset in the $\mathrm{BB}$ rat model of autoimmune diabetes expresses neither CD25 nor Foxp3," Journal of Immunology, vol. 177, no. 11, pp. 7820-7832, 2006.

[27] J. P. Mordes, R. Bortell, E. P. Blankenhorn, A. A. Rossini, and D. L. Greiner, "Rat models of type 1 diabetes: genetics, environment, and autoimmunity," ILAR Journal, vol. 45, no. 3, pp. 278-291, 2004.

[28] A. A. Rossini, E. S. Handler, J. P. Mordes, and D. L. Greiner, "Human autoimmune diabetes mellitus: lessons from BB rats and NOD mice-Caveat emptor," Clinical Immunology and Immunopathology, vol. 74, no. 1, pp. 2-9, 1995.

[29] B. Langeveld, A. J. M. Roks, R. A. Tio et al., "Rat abdominal aorta stenting: a new and reliable small animal model for instent restenosis," Journal of Vascular Research, vol. 41, no. 5, pp. 377-386, 2004.

[30] J. Visser, F. Klatter, J. L. Hillebrands, A. Jansen, L. Vijfschaft, and J. Rozing, "Thymectomy should be the first choice in the protection of diabetes-prone BB rats for breeding purposes," Laboratory Animals, vol. 38, no. 4, pp. 371-375, 2004.

[31] B. C. Mazzachi, M. J. Peake, and V. Ehrhardt, "Reference range and method comparison studies for enzymatic and Jaffe creatinine assays in plasma and serum and early morning urine," Clinical Laboratory, vol. 46, no. 1-2, pp. 53-55, 2000.

[32] J. Iwata and O. Nishikaze, "New micro-turbidimetric method for determination of protein in cerebrospinal fluid and urine," Clinical Chemistry, vol. 25, no. 7, pp. 1317-1319, 1979.
[33] R. W. Luxton, P. Patel, G. Keir, and E. J. Thompson, "A micromethod for measuring total protein in cerebrospinal fluid by using benzethonium chloride in microtiter plate wells," Clinical Chemistry, vol. 35, no. 8, pp. 1731-1734, 1989.

[34] R. S. Schwartz, K. C. Huber, J. G. Murphy et al., "Restenosis and the proportional neointimal response to coronary artery injury: results in a porcine model," Journal of the American College of Cardiology, vol. 19, no. 2, pp. 267-274, 1992.

[35] A. J. Cohen, P. D. McGill, R. G. Rossetti, D. L. Guberski, and A. A. Like, "Glomerulopathy in spontaneously diabetic rat: impact of glycemic control," Diabetes, vol. 36, no. 8, pp. 944951, 1987.

[36] P. D. Winocour and L. Hryhorenko, "Spontaneous diabetes in BB Wistar rats causes small increases in the early proliferative response of smooth muscle cells in re-injured aortae," Experimental and Molecular Pathology, vol. 63, no. 3, pp. 161-174, 1995.

[37] H. C. Groenewegen, G. Onuta, M. Goris et al., "Nonbone marrow origin of neointimal smooth muscle cells in experimental in-stent restenosis in rats," Journal of Vascular Research, vol. 45, no. 6, pp. 493-502, 2008.

[38] G. Onuta, P. E. Westerweel, A. Zandvoort et al., "Angiogenic sprouting from the aortic vascular wall is impaired in the BB rat model of autoimmune diabetes," Microvascular Research, vol. 75, no. 3, pp. 420-425, 2008.

[39] M. Osada, S. Takeda, R. Ogawa, S. Komori, and K. Tamura, "T lymphocyte activation and restenosis after percutaneous transluminal coronary angioplasty," Journal of Interferon and Cytokine Research, vol. 21, no. 4, pp. 219-221, 2001.

[40] H. Ø. Andersen, B. F. Hansen, P. Holm, S. Stender, and B. G. Nordestgaard, "Effect of cyclosporine on arterial balloon injury lesions in cholesterol-clamped rabbits: T lymphocytemediated immune responses not involved in balloon injuryinduced neointimal proliferation," Arteriosclerosis, Thrombosis, and Vascular Biology, vol. 19, no. 7, pp. 1687-1694, 1999. 


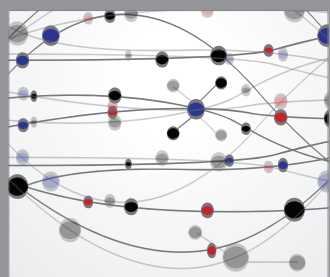

The Scientific World Journal
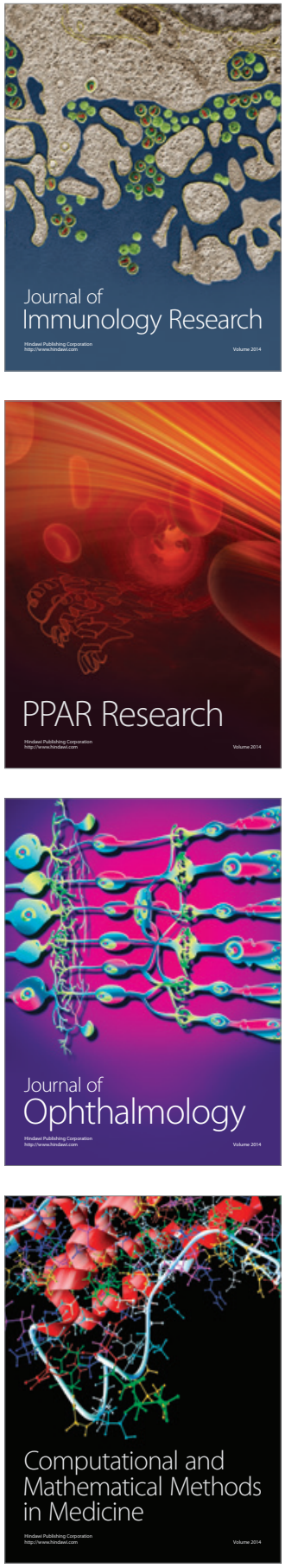

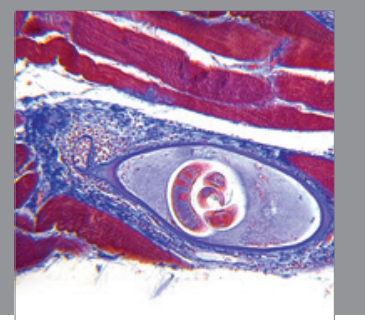

Gastroenterology

Research and Practice
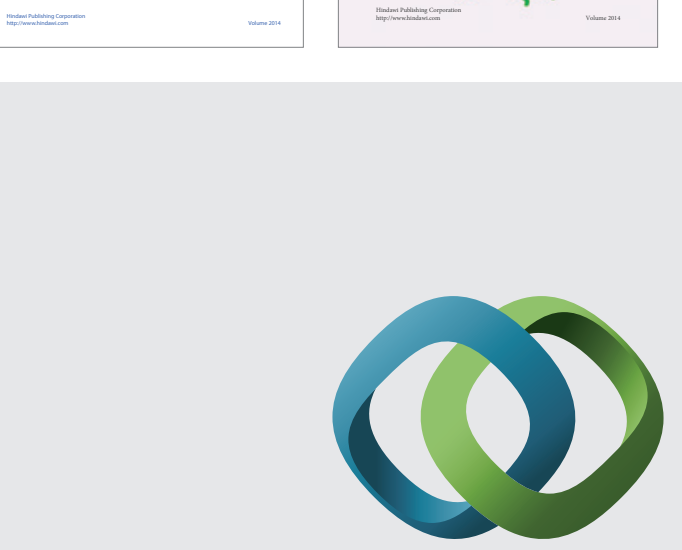

\section{Hindawi}

Submit your manuscripts at

http://www.hindawi.com
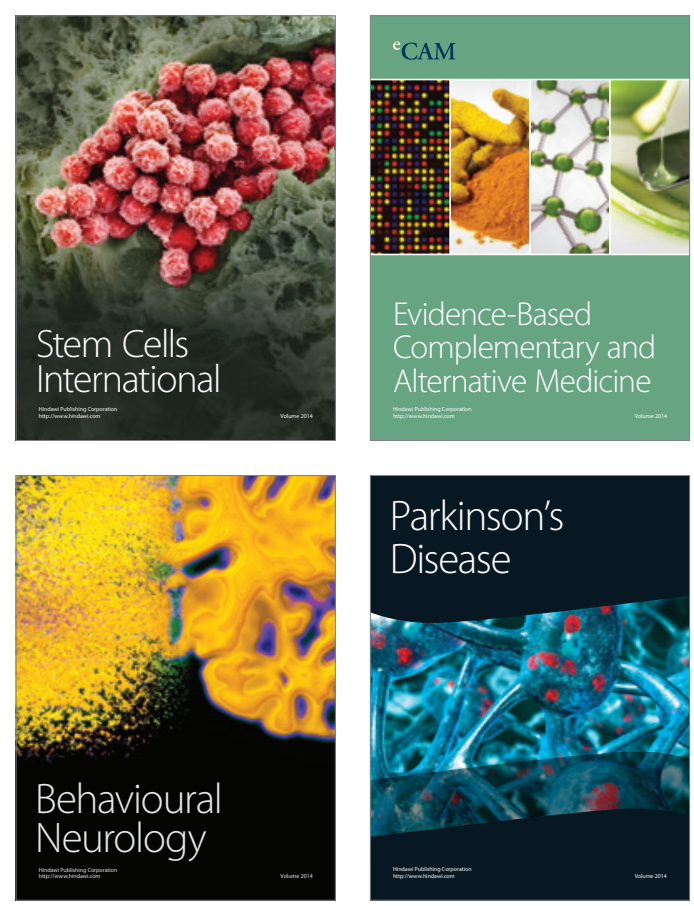

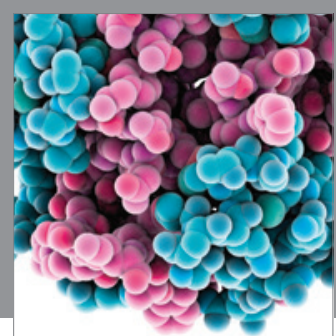

Journal of
Diabetes Research

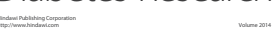

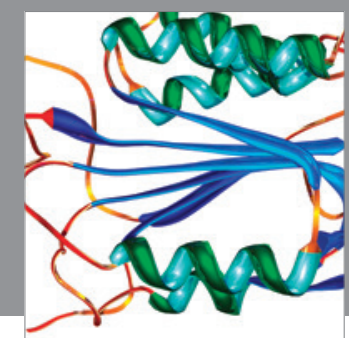

Disease Markers
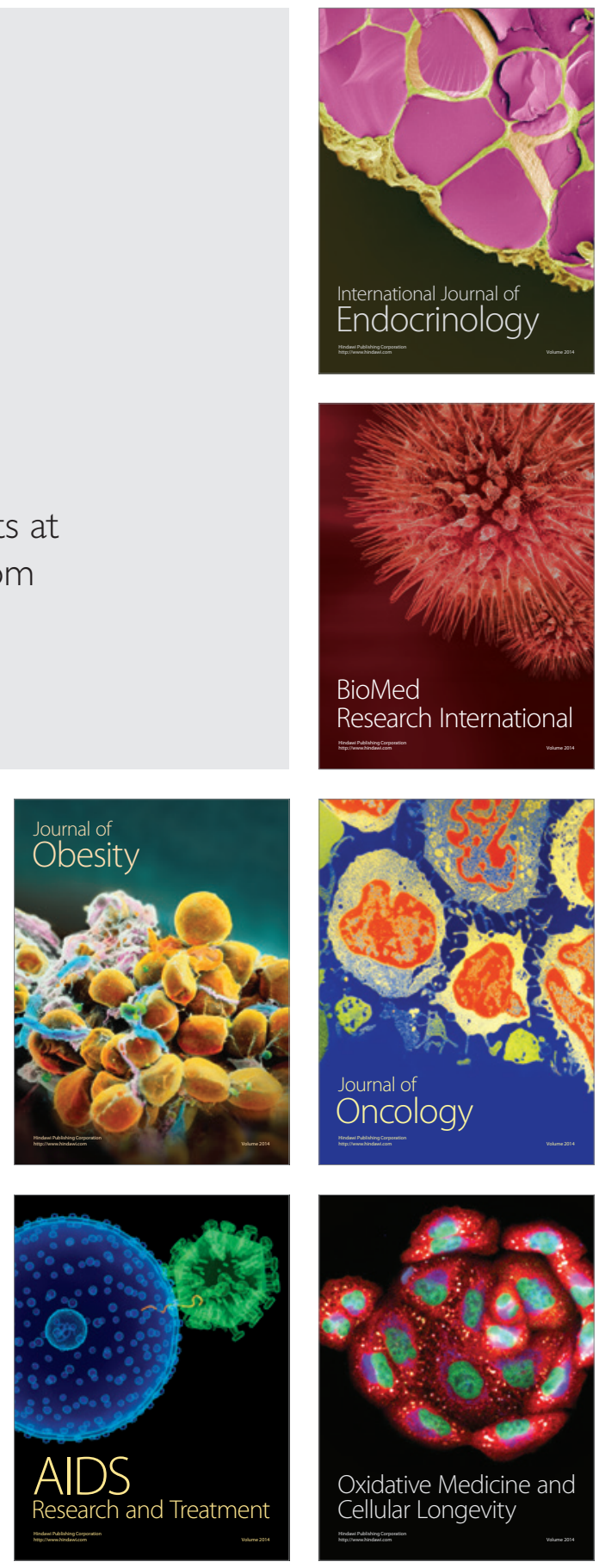